\section{Successful peroral endoscopic myotomy in situs inversus totalis}
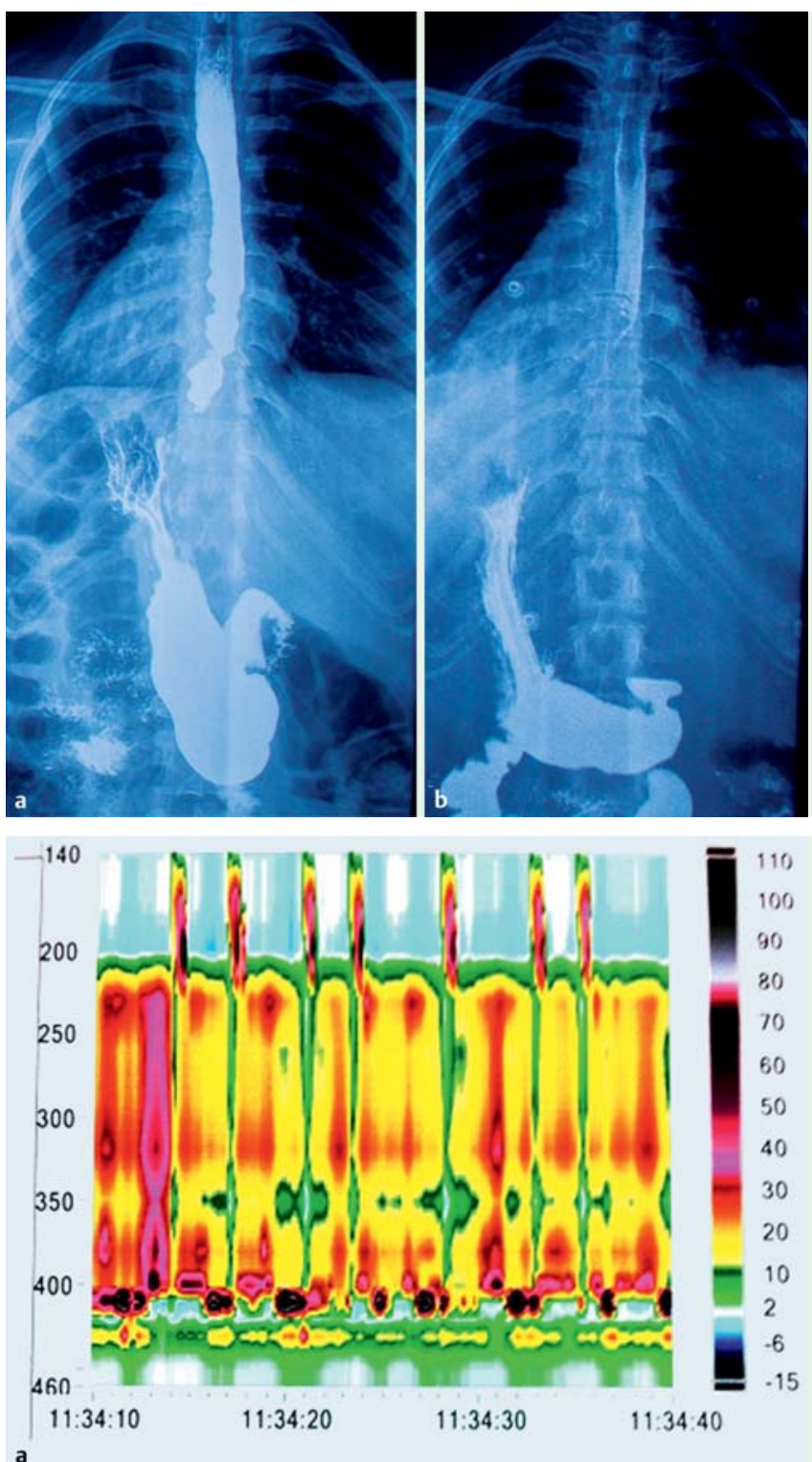

Fig. 2 High-resolution manometry. a Before peroral endoscopic myotomy (POEM), demonstrating esophageal aperistalsis and high basal lower esophageal sphincter (LES) pressure, with incomplete relaxation on swallowing and an integrated relaxation pressure $>15 \mathrm{mmHg}$. b At 5 weeks' follow-up post-POEM, showing normal basal LES pressure.

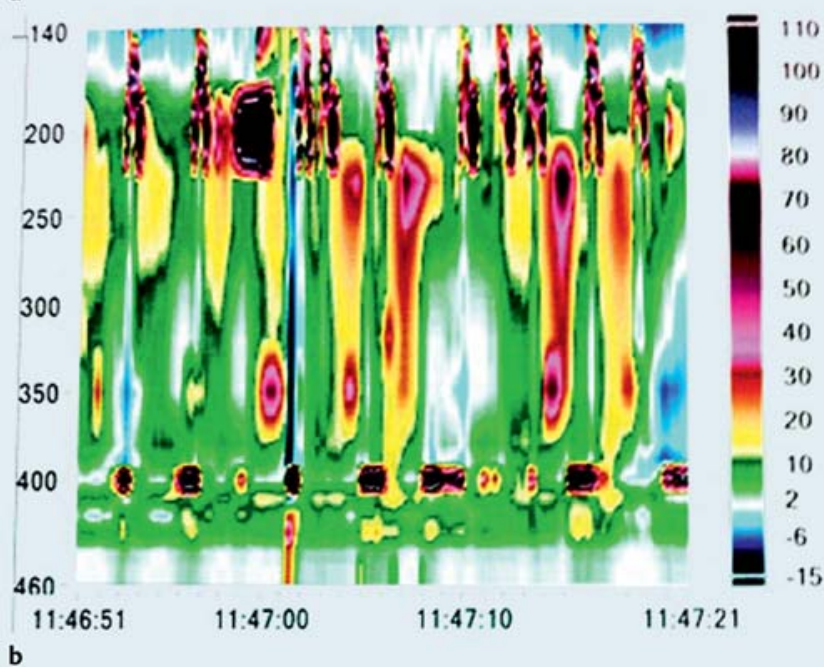

Situs inversus viscerum is a rare, autosomal, recessive condition in which the position of organs in the body is transposed to the opposite side. Most commonly, all viscera (abdominal and thoracic) are transposed, a condition known as situs inversus totalis (SIT) [1].

Endoscopic and surgical procedures in patients presenting with SIT require modification to the routine procedure, and this is also necessary for the newly described peroral endoscopic myotomy (POEM) for the treatment of achalasia [2]. We present a case of achalasia in a patient with SIT, who was successfully treated by POEM.

A 25-year-old woman with SIT presented with a 9-year history of achalasia, which had been treated by balloon dilation 4 years earlier. She still complained of dysphagia, weight loss, regurgitation, and pain (Eckardt score 9).

Esophagography ( Fig. 1 a) showed delayed esophageal emptying and narrowing at the lower esophageal sphincter (LES). High-resolution manometry demonstrated esophageal aperistalsis and high basal LES pressure with incomplete relaxation on swallowing ( Fig.2a). POEM was then performed with a few changes to the standard technique $[2,3]$.

The patient was placed in the supine position. As we prefer anterior myotomy, leading to the lesser gastric curvature, we started dissecting the esophagus segment at the 10 o'clock position, which is the opposite of the 2 o'clock position used in normal anatomy [2] ( Fig.3). Fluoroscopy guidance was helpful in this case. A 0.035 -inch radiopaque guidewire was coiled within the stomach of the patient as a radiological landmark while the submucosal tunnel was dissected. Once the esophagogastric junction had been reached, the endoscope was directed laterally towards the patient's right side. No other changes to the classically described POEM procedure [2] were necessary.

Postoperative esophagography revealed no leak of oral contrast and adequate passage into the stomach ( Fig. 1 b). At 5 weeks' follow-up, the patient's symptoms had resolved (Eckardt score 1), and basal LES pressure was normal ( $\bullet$ Fig. $\mathbf{2}$ b).

In conclusion, POEM is a feasible procedure in patients presenting with SIT, without the need for major changes to the technique.

Endoscopy_UCTN_Code_TTT_1AO_2AD

Competing interests: None 


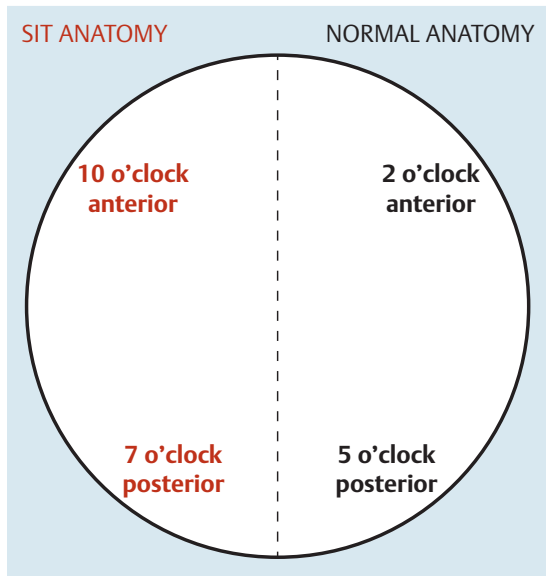

Fig. 3 Representation of the sites at which peroral endoscopic myotomy (POEM) is performed in supine position in normal anatomy (black, right part of the figure) and in situs inversus totalis (SIT) anatomy (red, left part of the figure). Anterior myotomy is performed at the 2 o'clock esophageal segment in normal anatomy and is performed at the 10 o'clock position in SIT. In contrast, posterior myotomy usually performed at the 5 o'clock position in normal anatomy, should be performed at the 7 o'clock direction in patients presenting with SIT.
Domenico Galasso ${ }^{1}$, Mohan Ramchandani' ${ }^{2}$, Rakesh Kalpala², Sundeep Lakhtakia², Rajesh Gupta², Santosh Darisetty $^{2}$, D. Nageshwar Reddy ${ }^{2}$

${ }^{1}$ Digestive Endoscopy Unit, “A. Gemelli” Hospital, Università Cattolica del Sacro Cuore, Rome, Italy

${ }^{2}$ Gastroenterology and Therapeutic Endoscopy Department, Asian Institute of Gastroenterology, Hyderabad, India

\section{References}

1 Gastrointestinal: situs inversus viscerum. J Gastroenterol Hepatol 2002; 17:

2 Inoue H, Minami H, Kobayashi Yet al. Peroral endoscopic myotomy (POEM) for esophageal achalasia. Endoscopy 2010; 42: $265-$ 271

3 Ramchandani M, Nageshwar RD. Peroral endoscopic myotomy: technique of mucosal incision. Clin Gastroenterol Hepatol 2014; 12: $900-901$
Bibliography

DOI http://dx.doi.org/ 10.1055/s-0034-1390848 Endoscopy 2014; 46: E648-E649

(c) Georg Thieme Verlag KG Stuttgart · New York ISSN 0013-726X

\section{Corresponding author} Mohan Ramchandani, MD

Gastroenterology and Therapeutic Endoscopy Department Asian Institute of Gastroenterology 6-3-661 Raj Bhavan Road Sangeet Nagar, Somajiguda Hyderabad Andhra Pradesh 500082 India

Fax: +91-40-23324255

ramchandanimohan@gmail.com 